\title{
Dietary fat and human cancer
}

\section{By M. J. Hill, Central Public Health Laboratory, Colindale Avenue, London $\mathrm{NW}_{9}$}

There are a number of 'cancers of western civilization' common in North-West Europe, North America and Australasia which are rare in Africa, Asia and South America. The incidence of these cancers in migrant populations from Japan, South-East Asia or Eastern Europe to North America or Australia is similar to that of their newly adopted home rather than to that of their country of origin, indicating that environmental factors are more important than genetic factors in determining the incidence of these diseases. Environmental factors can be divided into those associated with culture (e.g. diet, smoking and the use of other drugs, personal hygiene) and those associated with the physical environment (e.g. climate, air pollution, geographical location) and these can be dissociated by studying groups with different cultures living in the same city. When this is done it is found that in Bombay these cancers are more common in Christians and Muslims than in Hindus and Buddhists whilst in Johannesburg they are more common in white than in Indian or black people. From information of this sort it has been concluded that cultural factors, particularly diet, are of major importance in the aetiology of these cancers.

A relationship between diet and the incidence of a cancer may be due to the presence of preformed carcinogens or precarcinogens in the diet (as in, for example, the relation between dietary aflatoxin and liver cancer), or to an effect on the formation, activation or inactivation of carcinogens by the gut flora or by the tissue, or to an effect on the sensitivity of the tissues to carcinogens.

The cancers that I will discuss in this review are those of the large bowel, breast, endometrium, ovary and prostate. These all have the epidemiological characteristics described above and all except colo-rectal cancer are recognized to be hormone-dependent. All are associated with dietary fat when the diet and cancer incidences in large numbers of populations are correlated. In none of them is the association at all clear in case-control studies. They will be discussed in turn together with an indication of the current hypothesis on their causation and the possible mechanism of the association with dietary fat.

\section{Dietary fat and colon cancer}

Armstrong \& Doll (1975) demonstrated a strong correlation between dietary fat and colon cancer, confirming previous observations. Drasar \& Irving (1973) also showed that the correlation was better with animal than with vegetable fat and better with bound than with free fat. Many other dietary items have been correlated with colon cancer and these together with the epidemiology of colon cancer have been reviewed by Hill (1975), and the relation with dietary fat has been reviewed by Wynder \& Reddy (1975). 
The relationship between dietary fat and colon cancer is unlikely to be due to the presence of carcinogens in the diet. Many dietary carcinogens have been characterized but they are usually present in the greatest amounts in the diets of populations with a low incidence of colon cancer. The most popular mechanism involves the intestinal bile acids which are metabolized by the gut bacteria to a range of products which have been shown to be co-carcinogenic in animal studies and in the Salmonella mutagenesis assay of Ames. The faecal concentration of bile acids is increased with increased dietary fat, and further, it correlates with the incidence of colon cancer in studies of various populations around the world, in case-control studies and in studies of patient groups at increased risk of colon cancer. In animals treated with dimethylhydrazine (a carcinogen specific for the rodent large bowel) dietary changes or surgical manipulations which increase the faecal bile acid concentration increased the incidence of colon carcinomas and vice versa. The relationship between faecal bile acids and colon carcinogenesis has been reviewed by Hill (1977).

All colo-rectal carcinomas are thought to arise in pre-existing adenomas, and carriage of adenomas is thought to be determined by an autosomal recessive gene (Veale, 1965). Although the incidence of colon carcinomas is slightly higher in women than in men, the incidence of adenomas is twice as high in men as in women (Berge et al. 1973); thus a colonic adenoma is twice as likely to become malignant in a woman as in a man. Most carcinomas are more common in men than in women, indicating a sex-difference in the predisposition to carcinogenesis. The sex ratio for colon adenomas is similar to that for malignant tumours at many other sites (e.g. bladder, pancreas, stomach, kidney, oesophagus). Clearly the abnormality in colon cancer lies in the special predisposition of colonic adenomas to become malignant in women, and the demonstration of oestrogen binding sites in the colon indicates a possible role for oestrogens in carcinogenesis, providing a common link with cancers of the breast, endometrium and ovary.

In summary, there is no simple relation between dietary fat and colon cancer but hypotheses have been put forward that the mechanism depends on the effect of diet on the production of co-carcinogenic bacterial metabolites and on some hormonal effects.

\section{Dietary fat and endometrial cancer}

Armstrong \& Doll (1975) demonstrated a high correlation between total dietary fat and the incidence of endometrial cancer in twenty-three countries. The epidemiology of endometrial cancer has been reviewed by McMahon (1974) who showed its relation to obesity, early menarche, late menopause, hypertension, oestrogen therapy and previous cancer of the breast or ovary. In addition, it is associated with previous colon cancer or with colon cancer in the so-called 'cancer families'.

Armstrong (1977) has attempted to produce a unifying hypothesis to relate all of these to dietary fat intake. He has suggested that excess dietary fat causes excessive endogenous production of oestrogen and that this is the cause of 
endometrial cancer. A causative role of oestrogens in endometrial cancer is indicated from studies of the effects of oestrogen replacement therapy (Mack et al. 1976). The other observed correlations are secondary to this main one. Excess dietary fat is a cause of obesity (via its relation to total energy intake), of early menarche, and late menopause (both of which are related to total body-weight and obesity) and of hypertension (discussed by Armstrong, 1977). Breast cancer is strongly associated with excess dietary fat and so is likely to be coincidentally correlated with endometrial cancer. In addition, a role for oestrogens in breast cancer of the ovary but a role for oestrogens is strongly indicated, as will be discussed later.

Separating environmental and genetic causes is always very difficult. Studies of second cancers have indicated an association between cancer of the endometrium and cancer of the colon, indicating either common environmental or genetic factors. Both cancers are strongly correlated with dietary fat intake and this has been assumed by many to be the common factor. However, in his studies of 'cancer families' with a very high incidence of colon cancer Lynch et al. (1977) has noted that the females in the families also have a very high incidence of subsequent endometrial cancer, indicating a genetic association. It remains to be determined whether this association is limited to these families or is an indication of a general role for common genetic factors in these two cancers.

\section{Dietary fat and breast cancer}

A strong correlation between dietary fat and breast cancer incidence has been demonstrated by many groups, including Armstrong \& Doll (1975), Drasar \& Irving (1973), Carroll et al. (1968) and de Waard (1975). The general epidemiology of breast cancer has been reviewed by McMahon et al. (1973), and de Waard (1975).

Cancer of the breast is associated with obesity, is less common in women whose age at first pregnancy was low, is more common in women eating a high fat western diet than in Asian or African women, is associated with cancer of the endometrium, ovary and colon geographically and in persons with multiple primary carcinomas and is associated with a high urinary oestrogen:androgen ratio. The aetiology of breast cancer in premenopausal women appears to differ from that in post-menopausal women. The latter appears to be associated with diet whilst the former is less clearly so. A proportion of post-menopausal breast cancers appear to be oestrogen-dependent. There is no evidence that the association between post-menopausal breast cancer and diet is due to ingested pre-formed carcinogens or co-carcinogens and the generally accepted hypothesis is that it is due to an effect on endogenous production of carcinogens together with an effect on tissue susceptibility to carcinogens. A proportion of breast cancers is known to be oestrogen-dependent and oestrogen production is known to be related to obesity and to the amount of body fat (Lipsett, 1975) which itself is related to the amount of excess dietary fat and energy. Oestrogen-dependent tumours contain large numbers of oestrogen receptors (which are present in all breast tissue but at higher 
levels in malignant tissue). This observation has been used to determine the best course of treatment of breast cancer since only those with a high density of oestrogen receptors are likely to respond to ablation therapy to remove the sources of oestrogen (Jensen, 1975). The density of oestrogen receptors is thought to be related to breast adiposity which itself is related to dietary fat.

Although the picture is far from clear cut in humans, Carroll has obtained good evidence from animal studies supporting a role for dietary fat in breast carcinogenesis and that this is a tumour promoting effect rather than tumour initiation (Carroll, 1975). The effect was independent of energy intake (high-fat diets tend also to be high-energy diets).

In a number of studies of persons with second primary carcinomas there was a high association between endometrial and breast cancer. This could be due to a common relationship with obesity, to a common role for oestrogens in the carcinogenesis or to a common relationship with excess dietary fat.

\section{Dietary fat and cancer of the ovary and prostate}

Armstrong \& Doll (1975) showed dietary fat to be strongly correlated with the mortality from ovarian cancer but less so with its incidence (although it is still the dietary factor best correlated). The epidemiology of ovarian cancer has been reviewed by Lingeman (1974). Although this revealed an association with cancer of the breast, and that ovarian cancer is rare before menarche (although serious tumours are common in teenagers and young women) few real clues to the aetiology of this cancer were revealed. Ovarian cancers are hormone-dependent but the nature of the dependency is unclear. In the absence of any postulated aetiology of ovarian carcinogenesis the role of dietary fat in its causation must remain to be determined.

The situation is very similar with cancer of the prostate, in which there has been very little recent interest. Cancer of the prostate is also hormone-dependent in that it regresses following orchidectomy or as a result of treatment with oestrogens. The epidemiology of the disease has been reviewed by Wynder et al. (1971). Armstrong \& Doll (1975) found that dietary fat was highly correlated with mortality but not with incidence of prostatic cancer. This is almost certainly because prostatic cancer is very poorly diagnosed in general and incidence figures are notoriously unreliable. In view of the lack of any hypothesis on the aetiology of prostatic cancer there are no clues to the mechanism of the association with dietary fat.

\section{Conclusions}

Apart from the relation between lung cancer and cigarette smoking and the relationship between bladder cancer and industrial exposure to dyes, there are very few statements on cancer causation that can be made with any confidence. Claims regarding the role of diet are more notable for the messianic certainty of their proponents than for the strength of the evidence in their favour; dietary fat is not an exception to this. 
Further, if it is established that, for example, a diet rich in dietary fat causes colo-rectal cancer we must then examine carefully the likely effect of dietary manipulation on the risk of other cancers. For example, gastric cancer is associated with a low fat diet and we must be careful that we do not make recommendations which result in the incidence of a cancer with a relatively good prognosis (such as large bowel or endometrial cancer) being decreased whilst that of one with a poor prognosis (e.g. gastric cancer) is increased.

In my opinion there are at present no grounds for making recommendations, on the basis of a supposed role in carcinogenesis, designed to persuade us to give up or modify the diet that we like and have freely chosen.

The work of my laboratory is supported by the Cancer Research Campaign to whom the author would like to express thanks.

\section{REFERENCES}

Armstrong, B. K. (1977). In Origins of Human Cancer, p. 557 [H. Hiatt, J. Watson \& J. Winsten, editors]. New York: Cold Spring Harbor Press.

Armstrong, B. K. \& Doll, R. (1975). Int. F. Cancer 15, 617.

Berge, T., Ekelund, G., Mellner, C., Pihl, B. \& Wenckert, A. (1973). Acta chir. scand. supplement 438.

Carroll, K. K. (1975). Cancer Res. 35, 3374.

Carroll, K. K., Grammall, E. B. \& Plunkett, E. R. (1968). Can. med. Ass. F. 98, 590.

de Waard, F. (1975). Cancer Res. 35, 335I.

Drasar, B. S. \& Irving, D. (1973). Br. F. Cancer 27, 167.

Hill, M. J. (1975). C.R.C. Crit. Rev. Toxicol. 4, $3^{1}$.

Hill, M. J. (1977). In Origins in Human Cancer, p. 1640 [H. Hiatt, J. Watson and J. Winsten, editors]. New York: Cold Spring Harbor Press.

Jensen, E. V. (1975). Cancer Res. 35, 3362.

Lingeman, C. H. (1974). F. natn. Cancer Inst. 53, 1603.

Lipsett, M. (1975). Cancer Res. 35, 3359.

Lynch, H. T., Harns, R. E. \& Organ, C. H. (1977). Ann. Surg. 185, 435.

Mack, T. M., Pike, M. C., Henderson, B. E., Pfeffer, R. I., Gerkins, V. R., Arthur, M. \& Brown, S. E. (1976). NEngl. F. Med. 294, 1262.

McMahon, B. (1974). Gynec. Oncol. 2, 122.

McMahon, B., Cole, P. \& Brown, J. (1973). F. natn. Cancer Inst. 50, 21.

Veale, A. M. O. (1965). Eugenics Lab. Memoirs Series 40. London: Cambridge University Press.

Wynder, E. L., Mabuchi, K. \& Whitmore, W. F. (1971). Cancer 28, 344.

Wynder, E. L. \& Reddy, B. S. (1975). F. natn. Cancer Inst. 54, 7. 